\title{
Multi-Layered Effects of Fe on EMI Shielding of Sn-Al Hotel Architectural Powder
}

\author{
Fei-Shuo HUNG ${ }^{*}$ \\ Department of Travel Industry Management, Far East University, Tainan, Taiwan.
}

\begin{abstract}
No evident effect in shielding efficiency is observed when the electromagnetic wave-absorbing coating materials were applied in single layers because of the dispersing nature of the powder. When increased to two-layer coating, shielding effects were evident at both high and low frequencies, with greater shielding efficiency at low frequencies over high frequencies. It is worth noting that when increased to three-layer coating, as the weight percentage of powdered $\mathrm{Fe}$ increased from $5 \%$ to $8 \%$, the shielding efficiency of the powdered-Fe composite material was raised to $-35 \mathrm{~dB}$ This shows that, as the weight percentage gets higher, the powder shows the resonance phenomenon of permeability spectrum, and at high frequencies, the electromagnetic wave shielding efficiency of the composite materials were greatly increased. As the weight percentage of the powered $\mathrm{Fe}$ were increased to $8 \%$, we were unable to spread the powder evenly in the epoxy because of the dispersing characteristic in the electromagnetic properties of $\mathrm{Fe}$ and the anisotropic and heterogeneous nature of a powered composite material. During production, the powder aggregates often resulted in greater heterogeneity in the materials and consequently, lowered shielding efficiency at $3 \mathrm{GHz}$.
\end{abstract}

\section{Introduction}

Iron plays an important role in building materials; the ferrite magnet in the heavy metal ions of iron oxide are embedded in a stable state in the lattice [1-2]. The stability of the solid solution which in heavy metals is not easily perturbed and the chemical stability of heavy metals can be prevented. In addition to addressing environmental pollution [3-6],

\footnotetext{
*Corresponding Author:fshung@cc.feu.edu.tw
} 
the excellent magnetic properties of iron [7], the associated adsorption, conductivity[8], cost-competitiveness and EMI shielding and other potential applications are some of the advantages [9].

\section{Experimental Design}

In this study, the samples consisted of Sn-40Al-xFe ( hereafter referred to as SAF) ( $\mathrm{x}=$ $5,8$ wt. $\%, \mathrm{Fe}$ in the control of particle size $\sim \pm 30 \mu \mathrm{m})$ powder for performing EMI experiments. Appropriate amount of polypropylene resin (polypropylene) and SAF. powders were mixed while stirring ( $25 \mathrm{wt} . \%$ polypropylene ), so that the SAF powder is completely dispersed in the polypropylene resin. After stirring the Sn-Al-Fe mixed with polypropylene resin, the composite colloid was coated on a glass substrate (glass substrate size $2 \mathrm{~cm} \times 2 \mathrm{~cm} \times 2 \mathrm{~cm}$, total 4 ), and allowed to cure at room temperature for 24 hours. The coating thickness was controlled to $50 \pm 10 \mu \mathrm{m}$. Scanning electron microscopy was performed in order to characterize the morphology, microstructure and powder size of the various samples before and after baking. The obtained results were correlated with the evolution of the electromagnetic wave shielding related properties .The Elgal set 19A coaxial holder was used for electromagnetic interference shield testing. In this study, we implemented the coaxial EMI shielding effect apparatus, which is able to scan a large range of frequency of $300 \mathrm{~K}$ to $3 \mathrm{G} \mathrm{Hz}$, while the accuracy was controlled to within $10 \mathrm{ppm}$ $(250 \pm 50)$. The experiment was conducted by measuring the wavelength corresponding to perpendicular projection, while the frequency range was controlled in between $50 \mathrm{MHz}$ and $3000 \mathrm{MHz}$. Meanwhile, the results of each measurement were averaged 3 to 7 points, and the effective shielding rate was observed by performing further calculations [10-11].

\section{Results and Discussion}

\subsection{The Effects of Sn-xAl-Fe Coating Structure and Characteristics}

The first step was to mix Sn-40Al-xFe powder with $25 \mathrm{wt} . \%$ polyethylene; the powder coating experiment shows that single coating materials exhibit no significant shielding efficiency because the dispersion of the powder is too large. From previous studies, the expected number of layers of coating in the experiment was two to three and the 
thickness of each coat should be controlled to within $30 \pm 10 \mu \mathrm{m}$. $\circ$ The coating surface morphology for the Sn-40Al-5Fe and Sn-40Al-8Fe is shown in Fig. 1 (a) and Fig. 1 (b).It can be seen from the surface coating of the Sn-xAl sample that the Sn-40Al-8Fe powder has a large particle size and a loose structure in the mixing process, due to the fully disperse thin coating, as well as the relatively low surface roughness as seen in Fig.1(c). Table 1 shows the data of the electromagnetic wave shielding when coated in two layers of Sn-40AI-5Fe. The frequency range was controlled from $50 \mathrm{MHz}$ to $3 \mathrm{GHz}$ and its accuracy is $\pm 10 \mathrm{ppm}\left(25^{\circ}\right.$ to $\left.5^{\circ}\right)$. Each datum was the average of at least $3 \sim 7$ test results.
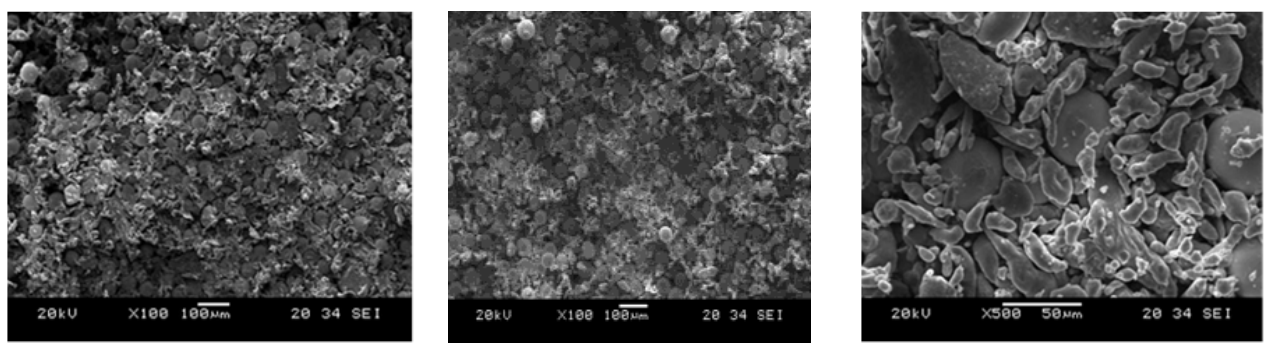

Fig. 1 SEM of Sn-xAl powders (a) Sn-40Al-5Fe ; SEM of (b) Sn-40Al-8Fe SEM of Enlarged Observation of $\mathrm{Sn}-40 \mathrm{Al}-8 \mathrm{Fe}(\mathrm{c})$

Table 1. Emi Shielding Effect Of Sn-40al-5fe Thin Film Under Different Db Value

\begin{tabular}{|c|c|c|c|c|}
\hline Specimen(-dB) & $300 \mathrm{MHz}$ & $900 \mathrm{MHz}$ & $1.80 \mathrm{GHz}$ & $3 \mathrm{GHz}$ \\
\hline $310 \mathrm{~nm}$ & -10.6710 & -15.8213 & -15.0626 & -15.7103 \\
\hline $720 \mathrm{~nm}$ & -21.3190 & -23.1781 & -22.3722 & -25.2142 \\
\hline $310 \mathrm{~nm}-\mathrm{H}$ & -9.1113 & -10.2723 & -12.8880 & -13.0877 \\
\hline
\end{tabular}

The corresponding $\mathrm{dB}$ value was constantly extracted for the comparison of EMI shielding effects. The results show that the coating is effective in shielding electromagnetic waves, regardless of the amount of Fe included. However, it is worth noting that the EMI shielding effect is most evident (compare Fig. 2 (a) and Fig. 2 (b) when the amount of Fe is increased (Fig. 2 (a)), and the effect is more prominent at high frequencies than at low frequencies. Therefore, it can be concluded that the electromagnetic wave shielding ability at high frequencies $(2.45 \mathrm{GHz})$ strengthen as the amount of Fe included is increased. Fig. 2 (b) shows the resulting effect of electromagnetic wave shielding with corresponding amount of Fe included in the coating. A comparison of Fig. 2 (a) and Fig. 2 (b) shows that 
the effect of EMI shielding is prominent regardless of the amount of Fe included. Regarding the Sn-40AI-5Fe coating, as the amount of Fe was increased to $8 \mathrm{wt} \%$, the EMI shielding of the sample became less effective at frequencies over $2.45 \mathrm{GHz}$ (Table.2).

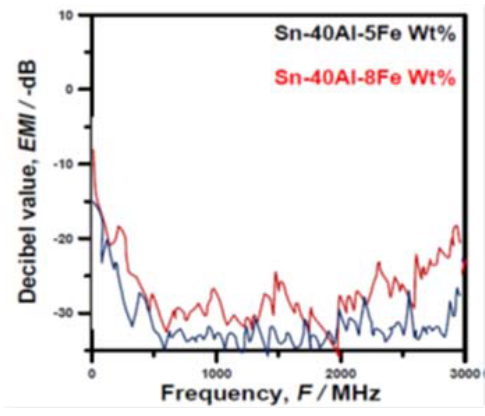

(a)

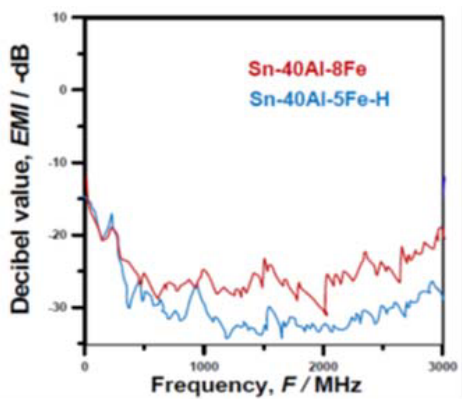

(b)

Fig.2 EMI shielding characteristics of Sn-Al-xFe Wt\%(a)\&(b) Fe content effect

Table 2. Emi Shielding Effect Of Sn-40al-5fe And Sn-40al-8fe Thin Film Under Different Db Value

\begin{tabular}{|c|c|c|c|c|}
\hline Specimen(-dB) & $300 \mathrm{MHz}$ & $900 \mathrm{MHz}$ & $1.80 \mathrm{GHz}$ & $3 \mathrm{GHz}$ \\
\hline Sn-40Al-8Fe -710nm & -12.2984 & -18.8346 & -22.9801 & -17.7783 \\
\hline Sn-40Al-8Fe -710nm-H & -10.2322 & -15.9891 & -17.5344 & -17.9597 \\
\hline Sn-40Al-5Fe -300nm & -10.6710 & -13.8111 & -12.2626 & -13.5103 \\
\hline Sn-40Al-5Fe-750nm & -21.2142 & -22.2437 & -16.9860 & -22.3414 \\
\hline
\end{tabular}

A comparison of the two samples, Sn-40AI-5Fe and Sn-40AI-8Fe (Fig. 2 (a) and Fig. 2 (b)), shows that the shielding effect is more prominent in Sn-40AI-5Fe at both high and low frequencies. In other words, the shielding ability of Sn-40AI-8Fe at high frequencies was not satisfactory, and it yielded no improvement even by adding layers. To sum it, the EMI shielding ability of Sn-40AI-xFe, at varying high frequencies, can be altered by changing the amount of Fe included. The Sn-40AI-5Fe sample is more effective in EMI shielding at both high and low frequencies. Based on earlier studies [25] and the results of the experiments, although the particles of the Sn-40AI-5Fe sample are larger in diameter, the loose nature of its structure improves its dispersibility, which contributes to the EMI shielding ability by forming a stacking effect upon coating. In regard to high frequencies, as the weight percentage of the powder was increased, the resonance phenomenon of permeability spectrum was observed, which greatly increased the shielding efficiency of the 
composite material at high-frequency of the electromagnetic waves. On the other hand, although $\mathrm{Sn}-40 \mathrm{Al}-8 \mathrm{Fe}$ is smaller in diameter, more compact, and has higher conductivity ( 6.18x10-5 (-cm)), its EMI shielding ability is inferior to Sn-40Al-5Fe. For Sn-40Al-5Fe, as the coating is increased from two layers to three layers, porosity is also increased to $\sim 18$ vol.\%, hence the decrease in conductivity. It is worth noting that we were unable to spread the powder evenly in the epoxy because of the dispersing characteristic in the electromagnetic properties of $\mathrm{Fe}$ and the anisotropic and heterogeneous nature of a powered composite material. During production, the powder aggregates often, resulting in greater heterogeneity in the materials, which is why the electromagnetic shielding ability does not increase along with the added layers of coating. Furthermore, Sn-40Al-5Fe coating is less costly to produce than Sn-40Al-8Fe. Additionally, the superior performance of $\mathrm{Sn}-40 \mathrm{Al}-5 \mathrm{Fe}$ at all frequencies should be noted. It can be concluded that Sn-40Al-5Fe should prove to be beneficial in the application of surface coating on building material to provide EMI shielding.

\section{Conclusions}

As the amount of Fe included in the $\mathrm{Sn}-40 \mathrm{Al}-5 \mathrm{Fe}$ coating is increased, despite the decrease in conductivity, the electromagnetic wave shielding ability at low frequencies is improved due to the stacking effect. At high frequencies, the resonance phenomenon of permeability spectrum and the decreased volume fraction of intermetallic compound prove to be significant findings in the attempt to raising the electromagnetic wave shielding efficiency in composite materials. Fe proves to be an important element for the improvement of electromagnetic shielding, due to its excellent crystalline structure, fine plasticity and thermal conductivity.

\section{Acknowledgments}

The authors are grateful to the Department of Travel Industry Management, Far East University for the financial support.

\section{References}

1. Akihiko, I., Hidetoshi, E., Hidemi, N., Kouji, W., and Osamu, H., "An Experimental Study of a $\lambda / 4$ Wave Absorber Using a Frequency-Selective Surface," Microwave and 
Optical Technology Letters, 2001, 28, 321-323.

2. Choi, H.D., Shim, H.W., Cho, K.Y., Lee, H.J., and Park, C.S., "Electromagnetic and Electromagnetic Wave-absorbing Properties of the SrTiO3-epoxy Composite," J. Applied Polymer Sci., 1999, 72, 75-83.

3. Regolini J.L., Margail J., Bodnar S., Maury D., Morin C. , Selective epitaxial Si based layers and TiSi2 deposition by integrated chemical vapor deposition. Applied Surface Science, 1996, 100, 566-574.

4. Knott, E.F., Shaeffer, J.F., and Tuley, M.T., Radar Cross Section. London: Artech House, 1993.

5. Naumann V., Otto M., Wehrspohn R.B., Werner M., Hagendorf C. , Interface and material characterization of thin ALD-A12O3 layers on crystalline silicon. Energy Procedia, 2012. 27.312-318.

6. Salisbury, W.W., "Absorbent Body for Electromagnetic Waves," U.S. Patent 2, Oct, 1952 .

7. C. M. Chiang and C. M. Lai: Building and Environment, 2001, 37, 387-392.

8. N. Day, J. Skinner, E. Roman and S. G. Allen: The Lancet, 1999, 354, 1925-1931.

9. MacFarlane, G.G., "Radar camouflage research and development by the Germans," Tech. Report T,1945, 99, 101-121.

10. F. S. Hung, F. Y. Hung and C. M. Chiang, Crystallization and Annealing Effects of Sputtered Tin Alloy Films on Electromagnetic Interference Shielding, Applied Surface Science, 2011. 257, 3733-3738.

11. F.S. Hung, F.Y. Hung, C.M. Chiang, T.S. Lui, Mater. Trans. 2008, 49, 655-660. 